\title{
Pyogenic Epidural Abscess with Primary Erector Spinae Pyomyositis - Case Report
}

\author{
Srinivasan U.S. , Jagadeesh P.C. and Senthil Kumar G.
}

Department of Neurosurgery and Orthopaedic Surgery, MIOT Hospitals, 4/112 Mount Poonamalle Road, Manapakkam, Chennai - 600 089, India

\begin{abstract}
Introduction: Pyogenic epidural abscess associated with primary pyomyositis of erector spinae in an immunocompetent individual is extremely rare. Here we report such a case which has been successfully managed.

Materials and Methods: A 26 year old man presented with severe pain in the low back region radiating to both the legs of 3 weeks duration. He was initially suspected to have prolapsed lumbar disc and treated conservatively without relief of symptoms. He had low grade fever on admission with no obvious systemic source of sepsis. Examination revealed only a positive straight leg raising test on both the sides with paravertebral muscle spasm and tenderness over the lumbar region without any other neurological deficit.

Results: Laboratory results showed leucocytosis, neutrophilia, elevated erythrocyte sedimentation rate and C-reactive protein. Magnetic resonance imaging (MRI) of the spine with contrast revealed an extensive L3 -S1 epidural abscess with multiple loculated collection of pus in the erector spinae muscles suggestive of pyomyositis. Abscess from the lumbar paravertebral muscles was drained, L5 bilateral laminectomy was performed and extradural abscess was completely evacuated. Pain was completely relieved in the immediate post operative period. Culture revealed growth of staphylococcus aureus which was sensitive to most of the antibiotics except oxacillin. Patient was treated with intravenous vancomycin for 3 weeks which was followed by oral linezolid for 3 weeks combined with oral ofloxacin for 6 weeks. Follow up spinal MRI scan with contrast performed after 6 months was normal. There was no recurrence of symptoms even at the end of 7 months.

Conclusion: Pyogenic spinal infection associated with paraspinal muscle abscess must always be considered when back pain with leg pain is associated with fever, leucocytosis and elevated ESR. Staphylococcus aureus is the most common pathogen in these cases. Hematogenous spread of infection through the venous communication between the external vertebral venous plexus with the internal vertebral veins, resulting in simultaneous abscess formation in both the sites is the probable pathogenesis in our case. Early surgical drainage of the abscess from both the sites, with concurrent use of antibiotic therapy for 6 weeks based upon the sensitivity of the organism is required to achieve an excellent outcome.
\end{abstract}

Keywords: Epidural abscess, primary pyomyositis, staphylococcus aureus.

\section{INTRODUCTION}

Pyogenic extradural spinal abscess is an uncommon condition [1,2]. Pyomyositis is a pyogenic infection of the skeletal muscle that can lead to abscess formation $[3,4]$. Both commonly occurs in the tropics and the main predisposing factors being immunocompromised individuals with HIV and diabetes [1-5]. The diagnosis of combination of pyomyositis and extradural spinal abscess is difficult due to their vague clinical presentation and poor localizing signs. A delay in diagnosis may result in sepsis and death [1-4]. Pyomyositis of erector spinae muscles can lead to infection in the adjacent structures like spine [6]. We report a rare case of extradural abscess in the lumbar region with pyomyositis of erector spinae muscles in an immunocompetent individual.

*Address correspondence to this author at the 6 Hamsadwani Apartments, 20, $1^{\text {st }}$ Canal Cross, Gandhinagar, Adyar, Chennai - 600 020, Tamil Nadu, India; Tel: 91 - 44- 2440 5050; E-mail: ussrini@vsnl.net

\section{MATERIALS AND METHODS}

A 26-year-old man presented with low back pain of severe intensity which was radiating to both the legs for more than 3 weeks. There was progressive decrease in the walking distance. He was treated conservatively suspecting a lumbar disc prolapse but with no relief of symptoms during the above period. On admission he had low-grade fever with no obvious systemic source of sepsis. Examination revealed bilateral positive straight leg raising test [SLR] with paravertebral muscle spasm and tenderness over the lumbar region without any focal neurological deficit.

Laboratory results showed leucocytosis [29800 cells/ cu.mm], neutrophilia [86\%], elevated erythrocyte sedimentation rate [ESR $83 \mathrm{~mm} / \mathrm{hour}$ ] and C-reactive protein $[25 \mathrm{mg} / \mathrm{l}]$. His blood sugar, renal parameters and liver function tests were normal. Serum electrophoresis showed an increase in the gamma globulin. Urine examination was normal. Radiographs of the chest and spine were normal. Computed tomography $[\mathrm{CT}]$ of the abdomen showed right iliac and obturator lymphadenopathy and CT chest was normal except for D7 vertebral body hemangioma. 
Spinal Magnetic Resonance Imaging [MRI] with contrast showed an extensive epidural mass lesion compressing the thecal sac posterolaterally at L3 - S1 level. It was hypoin-

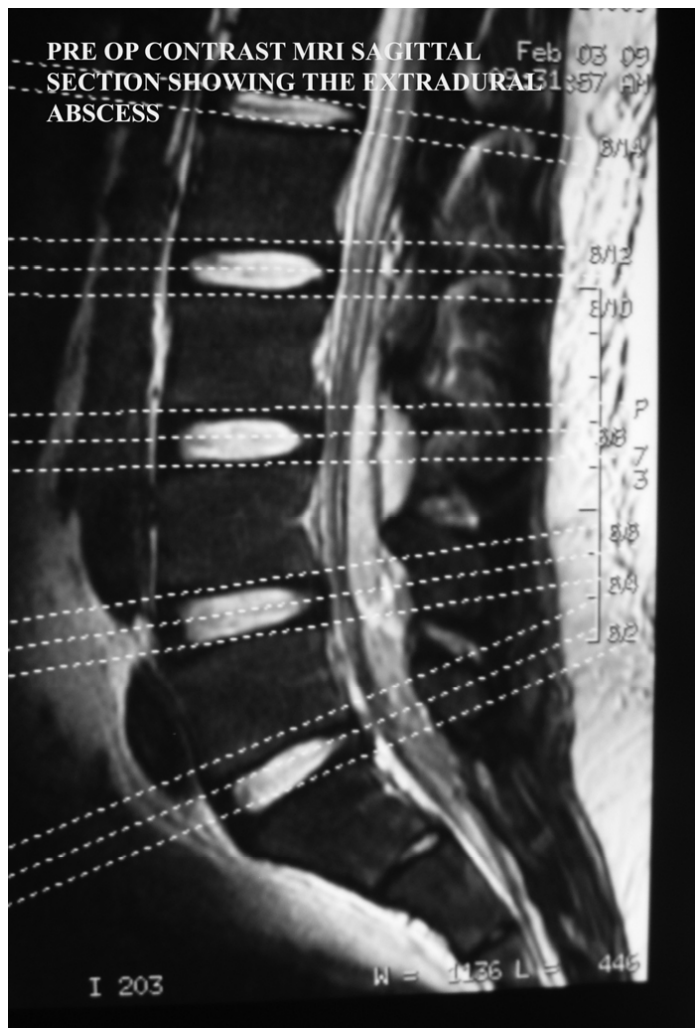

Fig. (1). Sagittal section Magnetic resonance imaging (MRI) showing the epidural lesion compressing the thecal sac in the lumbar region. tense in T1 and irregularly hyperintense in T2 images. Multiple abnormal signal regions were also noted in the posterior paravertebral muscles. Contrast study showed peripheral enhancement of the epidural lesion and the intramuscular paravertebral collections. The central portions of these lesions were non-enhancing indicative of fluid content or necrosis (Figs. 1 and 2). Areas of signal abnormality were demonstrated in adjacent bony structures but overt destructive process was not apparent. Psoas muscles were normal.

L3 - S1 was exposed, through the standard posterior approach. During subperiosteal separation of muscles, frank purulent liquid pus was observed in the paraspinal muscles on both the sides. Approximately $250 \mathrm{ml}$ of pus was drained from the paravertebral muscles. Pus from the intraspinal compartment, spontaneously drained through the interspinous space at L5 - S1. L5 spinectomy and bilateral laminectomy was performed and residual abscess in the lumbar and upper sacrum were drained. Pus sent for culture grew Staphylococcus aureus which was sensitive to most of the antibiotics except oxacillin. Patient was treated with intravenous vancomycin for 3 weeks which was followed by oral linezolid for 3 weeks combined with oral ofloxacin for 6 weeks. Patient pain in the lumbar region and both the legs, completely subsided within a week. By the end of 2 weeks, he was independently ambulatory without any neurological deficit. Follow up MRI scan of entire spine with contrast performed at the end of 6 months was normal (Fig. 3). There was no recurrence of symptoms even at the end of 7 months.

\section{DISCUSSION}

Pyogenic spinal extradural abscess is rare. Pyomyositis usually involves the large muscle groups of the pelvic girdle, lower extremities. It is of rare occurrence to involve only the

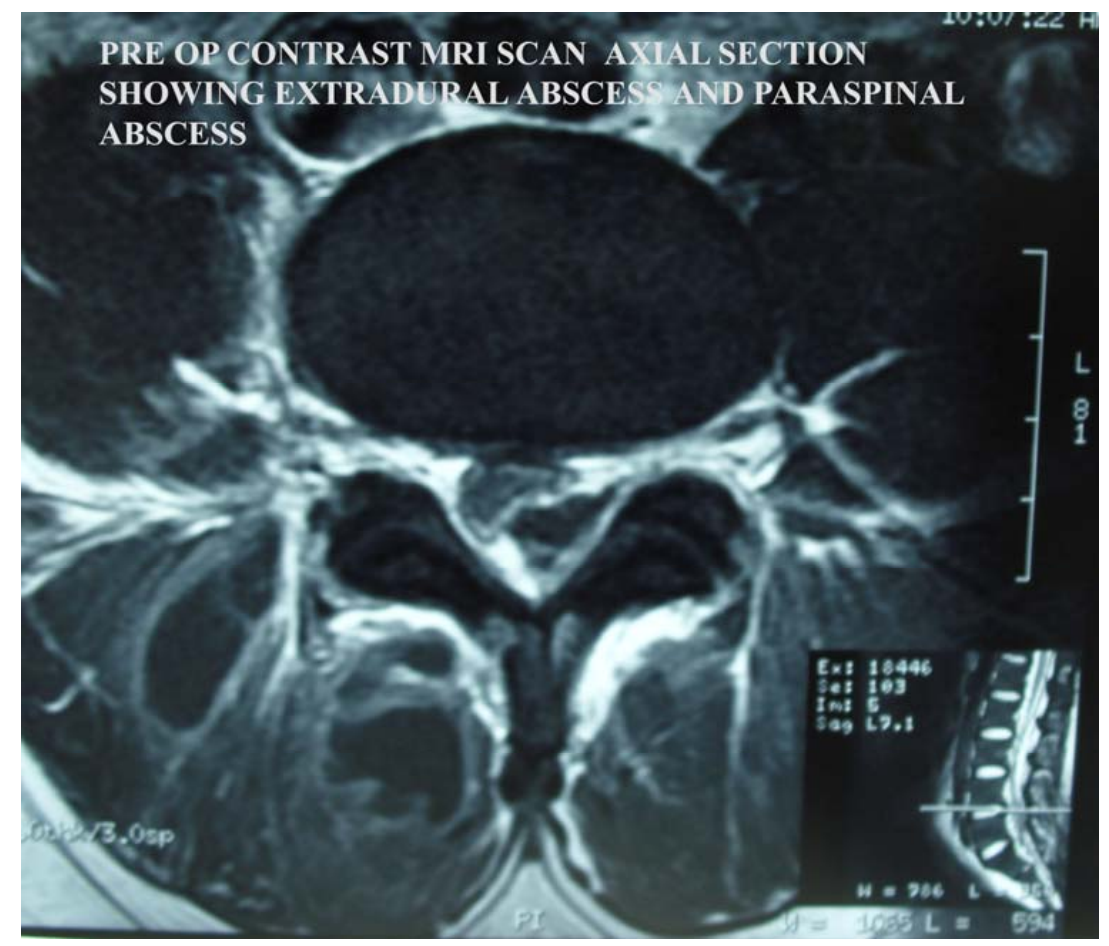

Fig. (2). Axial section Magnetic resonance imaging (MRI) showing the peripherally enhancing epidural abscess with multiple enhancing paravertebral abscesses. 
erector spinae muscles as seen in our case [6,7]. The combination of both these lesions in an immunocompetent person is extremely rare. Review of literature shows an isolated case report of pyomyositis with extradural abscess [6]. Staphylococcus aureus was the usual pathogen in both these lesions $[8,9]$. It may be 1-2 weeks before the correct diagnosis is made. If not treated early, toxicity and septic shock can occur, leading to death in both these conditions [15].

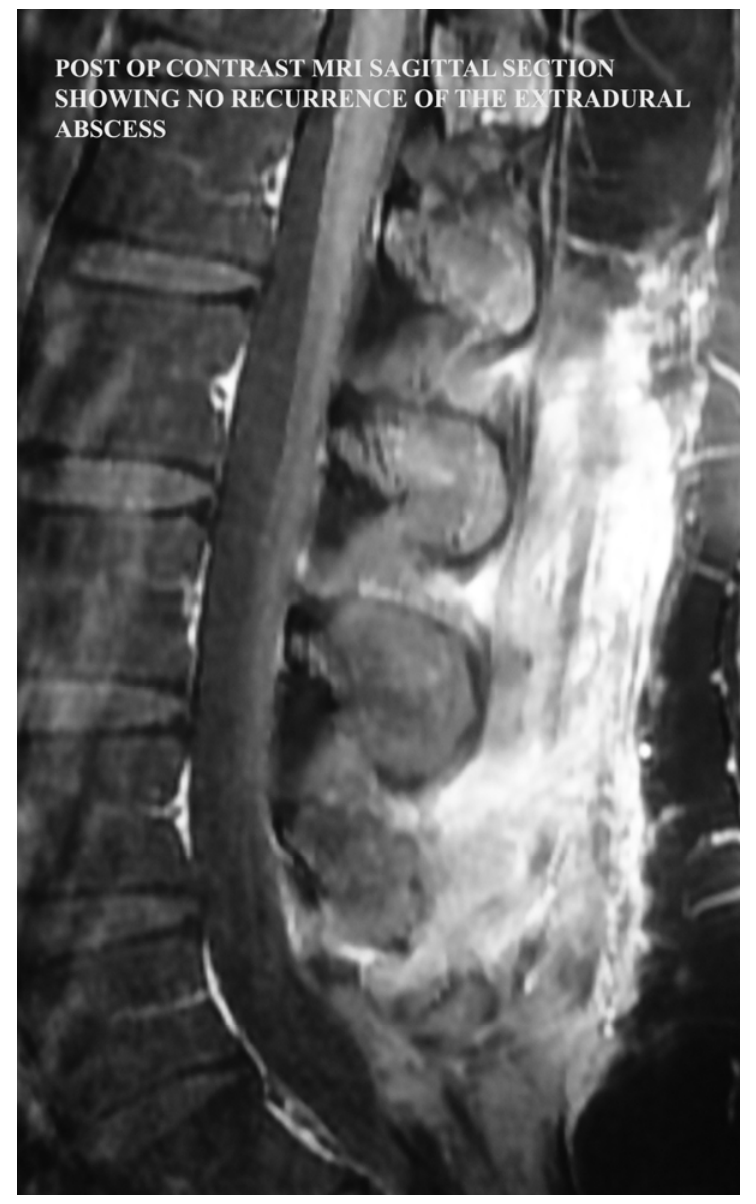

Fig. (3). Postoperative sagittal section Magnetic resonance imaging (MRI) showing no residual abscess.

The pathogenesis of pyomyositis is multifactorial. Transient bacteremia with concomitant muscle damage may be a causative factor. Pyomyositis progresses through three clinical stages of invasive phase, presuppurative phase and suppurative phase resulting in abscess formation [3-5]. While infection of the spinal epidural space results from hematogenous spread in $50 \%$ of cases $[10,11]$. It can also spread from local extension of infection from adjacent structures, many of which have been infected originally from hematogenous spread [11].

Correlating the above facts, we postulate that in our case, initially the triggering factor is the infection of the erector spinae muscles leading to pyomyositis. From this during the invasive phase of the pyomyositis, bacterimia of the posterior external plexus of veins which lie on the posterior surface of the lamina and around the spinous process would have occurred. These veins normally anastomose by passing through the ligamentum flavum with the internal vertebral venous plexus that lie within the vertebral canal between the duramater and the vertebrae [12]. Through these naturally existing venous communication, bacterimia of the internal vertebral veins should have taken place, leading to the development of posteriorly located epidural abscess at the same time. This hypothesis is supported by the fact that during surgery at L5- S1 level, after evacuation of the abscess located in the erector spinae muscles, there was spontaneous drainage of the extradural abscess from below the interspinous ligament of L5- S1. From both the sites, suppurative purulent pus was evacuated, indicating that both the abscess should have developed within the same period of time.

With abscess formation, surgical drainage of the purulent collection is required. This should be followed by concurrent use of antibiotic therapy for 6 weeks, based upon the sensitivity of the organism. Since staphylococcus aureus is the most common pathogen in both, extradural abscess [8] and pyomyositis [9], empirical treatment with flucloxacillin is appropriate but in our case we started him on vancomycin empirically and continued with it in the post operative period.

The diagnosis of pyogenic extradural spinal abscess and pyomyositis of erector spinae requires a high index of suspicion and should be considered in a patient with fever and local muscle pain. MRI with contrast is the imaging modality of choice. Successful treatment requires early recognition, appropriate use of antibiotic therapy with drainage of the abscess from the affected tissues.

\section{ACKNOWLEDGEMENT}

We wish to thank Prof. Dr. P.V.A. Mohandas, Managing Director MIOT Hospitals, Chennai, India for permitting us to do the above work.

\section{REFERENCES}

[1] Hernandez AG, Fenandez JC, Roman MP, Toledo VA, Gallego JH. Epidural spinal abscesses. Review of clinical series. Neurologia 2008; 23 (2): 85-90.

[2] Kempthorne JT, Pratt C, Smale EL, Macfarlane MR. Ten-year review of extradural spinal abscesses in a New Zealand tertiary referral center. J Clin Neurosci. 2009; 16 (8): 1038-42.

[3] Chiedozi LC. Pyomyositis: review of 205 cases in 112 patients. Am J Surg 1979; 137: 255-9

[4] Patel SR, Olenginski TP, Perruquet JL, Harringtom TM. Pyomyositis: clinical features and predisposing conditions. J Rheumatol 1997; 24: 1734-8.

[5] Seah MYY, Anavekar SN, Savige JA, Burrell LM. Diabetic pyomyositis. An uncommon cause of a painful leg. Diabetes care: 2004; 27 (7): 1743-4.

[6] Marshman LA, Bhatia CK, Krishna M, Friesem T. Primary erector spinae pyomyositis causing an epidural abscess: case report and literature review. Spine J 2008; 8(3): 548-51.

[7] Hassan FO, Shannak A. Primary pyomyositis of the paraspinal muscles: a case report and literature review. Eur Spine J 2008; 17 (2): 239-42.

[8] Chen WC, Wang JL, Wang JT, Chen YC, Chang SC. Spinal epidural abscess due to staphylococcus aureus: clinical manifestations and outcomes. J Microbiol Immunol Infect 2008; 41 (3): 215-21.

[9] Fan HC, Lo WT, Chu ML, Wang CC. Clinical characteristics of staphyloccal pyomyositis. J Microbiol Immunol Infect 2002; 35: 121-4. 
[10] Allen MB, Flunnery AM, Fisher J. Spinal epidural and subdural abscess. In: Wilkins RH, Rengachary SS, Eds. Neurosurgery. New York: McGraw-Hill 1996; pp. 3327-31.

[11] Zeidman SM, Ducker TB. Infectious complication in spine surgery. In: Benzel EC, Ed. Spine surgery, techniques, complication avoidance and management. New York: Churchill Livingstone, 1999; Vol. 1: pp. 1445-57.

[12] Williams PL, Warwick R. Veins of the thorax - Angiology. In: Gray's anatomy, $36^{\text {th }}$ ed. Edinburgh: Churchill Livingstone 1980; pp. 753-7.

(C) Srinivasan et al.; Licensee Bentham Open.

This is an open access article licensed under the terms of the Creative Commons Attribution Non-Commercial License (http: //creativecommons.org/licenses/by$\mathrm{nc} / 3.0 /$ ), which permits unrestricted, non-commercial use, distribution and reproduction in any medium, provided the work is properly cited. 